\title{
Análise das Complicações Hospitalares Relacionadas ao Cateterismo Cardíaco
}

\author{
Géderson Rossatoํ, Alexandre Schaan de Quadros¹, Rogério Sarmento-Leite1, \\ Carlos Antonio M. Gottschall ${ }^{1}$
}

\section{RESUMO}

Introdução: As complicações relacionadas ao cateterismo cardíaco são os principais limitantes desta técnica, e podem variar desde eventos adversos leves e transitórios até eventos graves, como infarto do miocárdio ou morte. Objetivo: Avaliar a incidência de complicações imediatas do cateterismo cardíaco diagnóstico em adultos, conforme um modelo de categorização das complicações em tipo e gravidade. Método: Estudo de coorte prospectivo em um centro de referência. As características dos pacientes foram registradas, e estes foram acompanhados até a alta hospitalar. As complicações foram categorizadas em nove modalidades: alérgica, isquêmica, vascular, arrítmica, vaso-vagal, pirogênica, neurológica, embólica e congestivas, e estratificadas em leves (intercorrências), moderadas (resolvidas em até 24 horas) ou graves (necessitou internação ou intervenção). Os fatores preditivos de complicações foram identificados por análise multivariada. Resultados: Foram incluídos 1916 indivíduos, sendo 59,4\% do sexo masculino e com média de idade de 58,3 $\pm 11,1$ anos. Complicações ocorreram em 175 pacientes (190 eventos), sendo que $62,5 \%$ foram intercorrências leves, $24 \%$ moderadas e $13,5 \%$ graves. Foi registrado apenas um óbito $(0,05 \%$ dos indivíduos). As complicações vasculares foram as mais incidentes $(35,6 \%)$, seguidas das vagais $(18,3 \%)$, isquêmicas $(15,4 \%)$ e alérgicas $(14,4 \%)$. Os fatores de risco para complicações pela análise multivariada foram uso de anticoagulante $(3,59 ; 1,67-7,74 ; p=0,006)$ e duração prolongada do exame $(1,03 ; 1,02-1,04 ; p<0,001)$. Conclusão: As complicações relacionadas ao cateterismo cardíaco são geralmente intercorrências sem gravidade, sendo que as complicações vasculares, reações vagais e isquêmicas foram as mais freqüentes. $\mathrm{O}$ uso de anticoagulantes e o tempo de exame prolongado foram os principais fatores preditivos de complicações.

DESCRITORES: Cateterismo cardíaco, efeitos adversos. Angiografia, efeitos adversos. Angiografia coronária, efeitos adversos.

\section{SUMMARY}

Analysis of In-Hospital Complications Related to Cardiac Catheterization

Background: The major limitations of cardiac catheterization are the occurrence of complications related to its invasive nature. Complications can vary from mild and transitory reactions to severe adverse events like myocardial infarction or death. Objective: To assess the incidence, type and severity of complications related to cardiac catheterization in adults using a comprehensive system previously described. Methods: We conducted a prospective cohort study in a tertiary reference center, and patient characteristics and in-hospital outcomes were registered in a dedicated database. Complications were categorized in nine types: allergic, ischemic, vascular, arrythmic, vagal, pyrogenic, neurological, embolic and congestive, and stratified in mild, moderate (resolved in 24 hours) or severe (needed hospitalization or another intervention). The predictive factors of complications were assessed by multivariate analysis. Results: We included 1916 individuals, 59\% male and with a mean age of $58 \pm 11$ years. Complications occurred in 175 patients (190 events), and $63 \%$ were mild, $24 \%$ moderate e $13 \%$ severe. There was one $(0.05 \%)$ death in the entire cohort. Vascular complications were the most common type $(36 \%)$, followed by vagal $(18 \%)$, ischemic $(15 \%)$ and allergic $(14 \%)$. Risk factors for complications by multivariate analysis were anticoagulant use $(\mathrm{OR}=3.59 ; \mathrm{Cl}=1.67-7.74 ; \mathrm{p}=0.006)$ and prolonged examination time $(\mathrm{OR}=1.03 ; \mathrm{Cl}=1.02-1.04 ; \mathrm{p}<0.001)$. Conclusions: In-hospital complications after cardiac catheterization are generally mild, and the events more commonly presented were vascular, vagal or ischemic. Anticoagulant use and prolonged examination time were independent predictors of complications.

DESCRIPTORS: Heart catheterization, adverse effects. Angiography, adverse effects. Coronary angiography, adverse effects.

Instituto de Cardiologia do Rio Grande do Sul / Fundação Universitária de Cardiologia, Porto Alegre, RS.

Correspondência: Unidade de Pesquisa do IC/FUC - Dr. Carlos Antonio M. Gottschall. Av. Princesa Isabel, 370 - Santana - Porto Alegre, RS - CEP 90620-001 - Fone/Fax: 51-3219-2802 Ramal 23,24 E-mail: editoracao-pc@cardiologia.org.br

Recebido em: 05/02/2007 - Aceito em: 15/03/2007 
A s complicações relacionadas ao cateterismo cardíaco são os principais limitantes desta técnica diagnóstica, e podem variar desde eventos adversos leves e transitórios até eventos graves, como infarto do miocárdio (IAM) ou morte ${ }^{1,2}$. A quantificação e qualificação dessas complicações podem ser realizadas de várias maneiras, sendo que Oliveira et al. ${ }^{3}$ descreveram um modelo classificatório abrangente e que leva em consideração o tipo da complicação e sua gravidade.

A comparação dos dados deste trabalho com os da literatura é necessária, pois, além de ser o único parâmetro disponível para a nossa prática diária, houve variações no perfil dos pacientes submetidos ao procedimento, na técnica de cateterismo, no material utilizado e evolução na curva de aprendizado dos Serviços de Hemodinâmica, desde os primeiros estudos até hoje.

O objetivo deste estudo foi verificar a incidência das complicações imediatas pós-cateterismo, aplicar e testar o modelo de complicações desenvolvido por Oliveira et al. ${ }^{3}$, comparando estes dados a outros modelos da literatura.

\section{MÉTODO}

\section{Pacientes}

Os pacientes incluídos neste estudo foram submetidos à cineangiocoronariografia, por indicação de seu médico assistente, no laboratório de hemodinâmica, no período compreendido entre 19/10/1999 e 9/08/2001. Os exames foram realizados em pacientes atendidos em nível ambulatorial ou internados, sendo os casos incluídos no estudo de forma consecutiva. O único critério de exclusão utilizado foi a recusa do pacientes em participar do estudo.

Os pacientes foram acompanhados clinicamente com o objetivo de avaliar as características demográficas e a ocorrência de complicações relacionadas ao procedimento desde sua internação até o momento da alta hospitalar. As características demográficas analisadas foram idade, sexo, peso, fatores de risco para cardiopatia isquêmica, diagnóstico clínico, presença de vasculopatia periférica, uso de anticoagulantes, duração do procedimento, quantidade de contraste, dados hemodinâmicos e diagnóstico angiográfico. Todos os pacientes assinaram termo de consentimento livre e esclarecido.

\section{Procedimentos}

Os procedimentos foram realizados por acesso femoral com cateteres $6 \mathrm{~F}$, pela técnica de Judkins ${ }^{4}$, ou por acesso braquial com cateteres 7 ou $8 \mathrm{~F}$, pela técnica de Sones ${ }^{5}$. Em pacientes com dificuldade de acesso, foram necessários ambos os acessos e técnicas, sendo considerado como técnica mista. A duração dos exames foi avaliada em minutos, desde o início da anestesia local até a retirada do último cateter. $\mathrm{O}$ contraste utilizado foi o diatrizoato de meglumina, sendo que o ioxitalamato foi utilizado em somente $0,63 \%$ dos pacientes, geralmente por indicação do operador, e em pacientes com história de alergia ou insuficiência renal.

O número de vasos com estenose maior ou igual a $70 \%$ e a presença de estenose significativa (>50\%) no tronco da artéria coronária esquerda foram registrados. A fração de ejeção foi avaliada por angiografia, conforme o método da área-longitude. A incidência de complicações foi também avaliada conforme o resultado do estudo hemodinâmico, normal ou patológico.

\section{Complicações}

As complicações foram classificadas em nove categorias, conforme a classificação de Oliveira et al. ${ }^{3}$ : alérgica, isquêmica, vascular, arrítmica, vaso-vagal, pirogênica, neurológica, embólica e congestiva. Em cada categoria, a complicação foi graduada conforme sua gravidade:

- Grau leve: intercorrência na sala de exame prontamente resolvida;

- Grau moderado: necessitou observação ou tratamento medicamentoso sem morbidade significativa e sem retardar a alta - resolvida em até 24 horas;

- Grau importante: necessitou internação ou intervenção exigindo tratamento intensivo ou intervencionista, nem sempre reversível, ou retardou a alta com morbi-mortalidade significativa ou causou seqüela.

A caracterização de cada categoria de complicação de acordo com sua gravidade está descrita no Quadro 1.

\section{Análise estatística}

Os dados foram expressos conforme suas médias e desvios-padrão. Variáveis contínuas ou categóricas foram analisadas pelo teste $t$ de Student, teste do qui quadrado ou teste exato de Fisher, sendo que a medida de associação entre as variáveis categóricas foi o risco relativo. Foi realizada análise multivariada por regressão logística múltipla para avaliação dos fatores de risco para complicações, sendo incluídas no modelo aquelas com $p<0,2$. Na análise univariada, foi adotado um nível de significância de $p<0,05$, com um intervalo de confiança de $95 \%$ para a análise univariada.

\section{RESULTADOS}

A amostra estudada foi de 1916 indivíduos, sendo $59,4 \%$ do sexo masculino, com média de idade de $58,3 \pm 11,1$ anos, e média de peso de $74 \mathrm{~kg}$. A Tabela 1 apresenta as características gerais da população, sendo que a média da fração de ejeção foi de $62,1 \pm$ $16,1 \%$, a média do volume de contraste foi de 98,0 $\pm 26,2 \mathrm{ml}(1,32 \mathrm{ml} / \mathrm{kg})$, e a duração do exame foi de 


\section{QUADRO 1 \\ Categorização das complicações e sua gravidade}

\begin{tabular}{|c|c|}
\hline Alérgica: & $\begin{array}{l}\text { 1. Leve: manifestações de pele } \\
\text { 2. Moderada: hipotensão, broncoespasmo reversível } \\
\text { 3. Importante: situações anteriores que evoluíram para choque e/ou óbito }\end{array}$ \\
\hline Isquêmica & $\begin{array}{l}\text { 1. Leve: angina controlada c/ nitrato } \\
\text { 2. Moderada: angina intensa e/ou evolui p/ edema agudo de pulmão ou baixo débito cardíaco } \\
\text { 3. Importante: revascularização de urgência, infarto ou óbito }\end{array}$ \\
\hline Vascular & $\begin{array}{l}\text { 1. Leve: hematoma ou sangramento pequeno, espasmo arterial } \\
\text { 2. Moderada: hematoma ou sangramento moderado, necessidade de reintervenção, espasmo arterial que } \\
\text { necessitou intervenção } \\
\text { 3. Importante: hematoma grande, sangramento intenso ou oclusão arterial com necessidade de cirurgia }\end{array}$ \\
\hline Arrítmica & $\begin{array}{l}\text { 1. Leve: taquiarritmia supraventricular, extrassistolia ventricular ou bradicardia sinusal } \\
\text { 2. Moderada: taquicardia ou fibrilação ventricular, assistolia prolongada } \\
\text { 3. Importante: necessidade de cardioversão elétrica e/ou marca-passo }\end{array}$ \\
\hline Vagal & $\begin{array}{l}\text { 1. Leve: náuseas, vômitos, sudorese ou palidez } \\
\text { 2. Moderada: bradiarritmia, hipotensão c/ necessidade de volume e/ou medicação } \\
\text { 3. Importante: quadro anterior que evolui para edema agudo de pulmão, infarto do miocárdio ou assistolia }\end{array}$ \\
\hline Pirogênica & $\begin{array}{l}\text { 1. Leve: hipertermia } \\
\text { 2. Moderada: bacteremia (hipertermia, cianose, calafrios e tremores) } \\
\text { 3. Importante: hipertermia com hipotensão ou choque }\end{array}$ \\
\hline Neurológica & $\begin{array}{l}\text { 1. Leve: sonolência, diplopia, tontura } \\
\text { 2. Moderada: manifestações parcialmente reversíveis } \\
\text { 3. Importante: manifestações irreversíveis }\end{array}$ \\
\hline Embólica & $\begin{array}{l}\text { 1. Leve: embolia periférica sem repercussão } \\
\text { 2. Moderada: embolia periférica ou central reversível } \\
\text { 3. Importante: embolia irreversível, óbito }\end{array}$ \\
\hline Congestiva & $\begin{array}{l}\text { 1. Leve: Killip I } \\
\text { 2. Moderada: Killip II } \\
\text { 3. Importante: EAP, choque cardiogênico }\end{array}$ \\
\hline
\end{tabular}

7 a $131 \min (23,8 \pm 11,2 \mathrm{~min})$. Em relação à técnica utilizada para realizar o procedimento, $1728(90,2 \%)$ casos foram realizados pela técnica de Judkins e 183 $(9,6 \%)$ pela técnica de Sones.

$\mathrm{Na}$ Tabela 2, verifica-se que em 175 pacientes foram registradas complicações do procedimento, sendo que alguns deles apresentaram mais de um tipo de intercorrência, perfazendo um total de 190 eventos (10,4\% dos procedimentos). Destas, 122 (6,5\%) foram leves, 47 (2,5\%) moderadas e 23 (1,4\%) importantes, havendo maior incidência das complicações vasculares $(3,7 \%)$, vaso-vagais $(1,9 \%)$, isquêmicas $(1,6 \%)$ e alérgicas $(1,5 \%)$. As complicações importantes ocorreram em somente 1,4\% dos pacientes, sendo $0,5 \%$ isquêmicas, $0,4 \%$ arrítmicas, $0,3 \%$ vasculares e $0,1 \%$ congestivas, além de $0,1 \%$ no grupo "outras" - não especificadas. Observamos apenas um $(0,05 \%)$ óbito decorrente do exame, devido a choque cardiogênico seguido de assistolia em um paciente com disfunção miocárdica grave por coronariopatia (lesão de três vasos e infartos prévios nos territórios das três artérias coronárias principais).

Quando comparado o grupo de procedimentos sem complicações com os casos complicados, não houve diferença estatisticamente significativa quanto a idade, sexo, peso, volume de contraste injetado e fração de ejeção. A presença de diabetes mellitus, tabagismo, lesão de tronco da coronária esquerda, cardiopatia isquêmica, uso de anticoagulante e aumento na duração do exame estiveram associados a um maior número de complicações, que também aumentaram proporcionalmente ao maior número de vasos com lesão $(p<0,05)$ - Tabela 3. No entanto, as únicas características que apresentaram associação independente com a presença de complicações foram o uso de anticoagulantes e tempo de execução do exame (Tabela 4). O uso de anticoagulantes foi associado com risco 3,59 vezes maior de complicações $(p=0,006)$, e o risco de complicações foi $3 \%$ maior a cada minuto excedido em tempo $(p<0,001)$. 
TABELA 1

Características da População Estudada (n=1916)

\begin{tabular}{lc}
\hline Característica & \\
\hline Gênero masculino, n (\%) & $1138(59,4)$ \\
Idade (anos) & $58,3 \pm 11,1(16-93)$ \\
Peso (kg) & $74,0 \pm 13,5(38-159)$ \\
Técnica, $\mathrm{n}(\%)$ & $1728(90,2)$ \\
$\quad$ Judkins & $183(9,6)$ \\
$\quad$ Sones & $4(0,2)$ \\
Mista & $62,1 \pm 16,1(14-99)$ \\
Fração de ejeção (\%) & $98,0 \pm 26,2(0-300)$ \\
Volume de contraste (ml) & $23,8 \pm 11,2(7-131)$ \\
Duração exame (minutos) & \\
Diagnósticos, $\mathrm{n}(\%)$ & $961(50,1)$ \\
$\quad$ Cardiopatia isquêmica & $184(9,5)$ \\
$\quad$ Valvulopatias & $54(2,8)$ \\
$\quad$ Miocardiopatias & $723(37,7)$ \\
$\quad$ Normais &
\end{tabular}

Variáveis contínuas = média \pm desvio-padrão (amplitude)

Variáveis categóricas $=$ número de pacientes (porcentual)

As características associadas às complicações isquêmicas na análise univariada foram uso de ácido acetilsalicílico (AAS), uso de heparina, hipertensão arterial sistêmica, lesão de tronco da artéria coronária esquerda e diagnóstico de cardiopatia isquêmica. Por análise multivariada, os preditores independentes de complicações isquêmicas foram Hipertensão arterial $(R C=3,00$ $[1,32-6,85] ; p=0,03)$, lesão de tronco de coronária esquerda $(R C=4,41[1,62-11,9] ; p=0,01)$ e cardiopatia isquêmica ( $R C=3,89$ [1,55-9,75]; $p=0,01)$.

Os fatores associados a complicações vasculares na análise univariada foram o uso de anticoagulante, pulso pedioso diminuído pré-exame, via de acesso braquial e maior tempo de exame, enquanto que o maior peso corporal foi fator protetor para este tipo de complicação. Por análise multivariada, somente uso de anticoagulante $(R C=3,40[1,25-9,22] ; p=0,04)$, acesso braquial $(R C=2,98[1,46-6,08] ; p=0,01)$ e maior duração do exame $(R C=1,04[1,03-1,06] ; p<0,001)$ foram independentemente associados a complicações.

\section{DISCUSSÃO}

A análise da prevalência das complicações e seus fatores predisponentes é uma preocupação constante de um Laboratório de Hemodinâmica, e, neste estudo, apresentamos os resultados de um protocolo de monitoramento e avaliação de complicações baseado no modelo descrito por Oliveira et al. ${ }^{3}$.

\section{Características da população e complicações}

Nesta série, foi registrada somente uma morte conseqüente ao cateterismo cardíaco esquerdo, que é inferior às estatísticas recentes, conforme demonstrado na Tabela 5. É importante salientar que alguns estudos incluíram pacientes submetidos à angioplastia coronariana $^{1,6,7}$, que está associada à maior mortalidade. A baixa mortalidade registrada em nosso estudo também pode estar associada à decisão de incluir somente exames diagnósticos e ao perfil de baixo risco, pois $38 \%$ dos exames foram considerados normais, sendo a maioria de pacientes ambulatoriais.

A média de idade e a prevalência de indivíduos do sexo masculino na população estudada foi semelhante a estudos anteriores ${ }^{6,8-12}$, e não estiveram associados a aumento global de complicações. No entanto, os pacientes com complicações moderadas e importantes apresentaram média de idade de 64 anos, confirmando relato de estudos prévios ${ }^{9,13}$.

TABELA 2

Incidência das Complicações por Categoria nos 1916 Casos Estudados

\begin{tabular}{lcccc}
\hline Categoria & Leve & Moderada & Importante & Total \\
\hline Vascular & $53(2,8)$ & $11(0,6)$ & $5(0,3)$ & $69(3,7)$ \\
Vaso-vagal & $19(1,0)$ & $17(0,9)$ & 0 & $36(1,9)$ \\
Isquêmica & $17(0,9)$ & $3(0,2)$ & $9(0,5)$ & $29(1,6)$ \\
Alérgica & $22(1,1)$ & $8(0,4)$ & 0 & $30(1,5)$ \\
Arrítmica & $3(0,2)$ & $4(0,2)$ & $8(0,4)$ & $15(0,8)$ \\
Pirogênica & $3(0,2)$ & $2(0,1)$ & 0 & $5(0,3)$ \\
Neurológica & $2(0,1)$ & $2(0,1)$ & 0 & $4(0,2)$ \\
Congestiva & 0 & 0 & $1(0,1)$ & $1(0,1)$ \\
Embólica & $1(0,1)$ & 0 & 0 & $1(0,1)$ \\
Outras & $1(0,1)$ & 0 & $24(1,4)$ & $2(0,2)$ \\
Total & $121(6,5)$ & $47(2,5)$ & $192(10,4)$ \\
\hline
\end{tabular}

Variáveis categóricas = número de pacientes (porcentual). 
TABELA 3

Comparação entre os grupos de pacientes com e sem complicações conforme características clínicas e angiográficas

\begin{tabular}{|c|c|c|c|c|}
\hline Característica & $\begin{array}{c}\text { Com complicação** } \\
n=175(9,1 \%)\end{array}$ & $\begin{array}{l}\text { Sem complicação } \\
\mathrm{n}=1741(90,9 \%)\end{array}$ & $\begin{array}{c}\text { Total }=1916 \\
n(\%)\end{array}$ & $\mathbf{p}$ \\
\hline Idade (anos) & 58,33 & 57,82 & & $0,56^{\&}$ \\
\hline Gênero (\%) & & & & $0,38^{\#}$ \\
\hline Masculino & $98(8,6)$ & $1040(91,4)$ & $1138(59,4)$ & \\
\hline Feminino & 77 & $(9,9)$ & $701(90,1)$ & $778(40,6)$ \\
\hline Peso (kg) & 74,08 & 72,87 & & $0,26^{\&}$ \\
\hline Total de contraste $(\mathrm{ml})$ & 97,77 & 100,20 & & $0,24^{\&}$ \\
\hline Diabetes mellitus (\%) & & & & $0,049^{\#}$ \\
\hline Presente & $36(12,4)$ & $255(87,6)$ & $291(15,2)$ & \\
\hline Ausente & $139(8,6)$ & $1486(91,4)$ & $1625(84,8)$ & \\
\hline Tabagismo (\%) & & & & $0,04^{\#}$ \\
\hline Presente & $91(8,0)$ & $1050(92,0)$ & $1141(59,5)$ & \\
\hline Ausente & $84(10,8)$ & $691(89,2)$ & $775(40,5)$ & \\
\hline Lesão de TCE (\%) & & & & $0,047^{*}$ \\
\hline Presente & $8(19,5)$ & $33(80,5)$ & $41(2,1)$ & \\
\hline Ausente & $167(8,9)$ & $1708(91,1)$ & $1875(97,9)$ & \\
\hline Cardiopatia isquêmica (\%) & & & & $0,007^{\#}$ \\
\hline Presente & $106(10,9)$ & $863(89,1)$ & $969(50,6)$ & \\
\hline Ausente & $69(7,3)$ & $878(92,7)$ & $947(49,4)$ & \\
\hline № de vasos (\%) & & & & 0,015 \\
\hline 0 & $70(7,3)$ & $885(92,7)$ & $955(49,8)$ & \\
\hline 1 & $43(11,2)$ & $342(88,8)$ & $385(20,1)$ & \\
\hline 2 & $30(9)$ & $303(91)$ & $333(17,4)$ & \\
\hline$\geq 3$ & $32(13,2)$ & $211(86,8)$ & $243(12,7)$ & \\
\hline Fração de ejeção (\%) & $59,7 \pm 17,4$ & $62,1 \pm 16,4$ & & $0,07^{\&}$ \\
\hline Tempo de exame (minutos) & $28,2 \pm 15,8$ & $23,4 \pm 10,5$ & & $<0,001^{\&}$ \\
\hline Uso de anticoagulante (\%) & & & & $0,013^{*}$ \\
\hline Presente & $7(24,1)$ & $22(75,9)$ & $29(1,5)$ & \\
\hline Ausente & $168(8,9)$ & $1719(91,1)$ & $1887(98,5)$ & \\
\hline
\end{tabular}

TCE = Tronco de Coronária Esquerda

Variáveis contínuas = média \pm desvio-padrão (amplitude). Variáveis categóricas = número de pacientes (porcentual)

TABELA 4 Análise multivariada das características clínicas e angiográficas associadas com complicações

\begin{tabular}{|c|c|c|c|c|}
\hline Característica & $\begin{array}{l}\text { Razão de chance } \\
\qquad(I C=95 \%)\end{array}$ & $\mathbf{p}$ & $\begin{array}{l}\text { Razão de chance ajustada } \\
\qquad(I C=95 \%)\end{array}$ & $\mathbf{p}$ \\
\hline Pulso pedioso diminuído & $1,74(0,87 ; 3,44)$ & 0,17 & $1,25(0,68 ; 2,28)$ & 0,54 \\
\hline Uso de AAS & $1,47(0,95 ; 2,27)$ & 0,096 & $1,51(1,00 ; 2,25)$ & 0,09 \\
\hline Uso de anticoagulante & $3,25(1,37 ; 7,73)$ & 0,013 & $3,59(1,67 ; 7,74)$ & 0,006 \\
\hline HAS & $1,27(0,92 ; 1,76)$ & 0,167 & $1,19(0,90 ; 1,60)$ & 0,30 \\
\hline DM & $1,51(1,02 ; 2,23)$ & 0,039 & $1,27(0,91 ; 1,80)$ & 0,24 \\
\hline Tabagismo & $1,40(1,03 ; 1,91)$ & 0,04 & $1,33(1,01 ; 1,75)$ & 0,09 \\
\hline Lesão de TCE & $2,48(1,13 ; 5,50)$ & 0,047 & $1,85(0,93 ; 3,67)$ & 0,14 \\
\hline Maior duração do exame & $1,03(1,02 ; 1,04)$ & $<0,001$ & $1,03(1,02 ; 1,04)$ & $<0,001$ \\
\hline Fração de ejeção & $0,99(0,98 ; 1,00)$ & 0,07 & $0,99(0,98 ; 1,02)$ & 0,20 \\
\hline Cardiopatia isquêmica & $1,56(1,13 ; 2,14)$ & 0,007 & $1,22(0,91 ; 1,63)$ & 0,26 \\
\hline
\end{tabular}

IC=Intervalo de confiança; AAS=Ácido Acetilsalicílico; HAS=Hipertensão Arterial Sistêmica; DM=Diabetes Mellitus; TCE=Tronco de Coronária Esquerda 
O tempo de duração do procedimento no grupo de pacientes com complicações foi significativamente maior do que aquele nos pacientes sem complicações, confirmando achados anteriores ${ }^{8,14}$. Embora tenha sido sugerido que a duração prolongada do exame não seja a causa das complicações, e sim um marcador da gravidade do paciente e de casos com maiores dificuldades técnicas, esta variável foi um preditor independente de complicações totais e vasculares na análise multivariada.

Os pacientes com disfunção sistólica importante (FE $<20 \%$ ) necessitaram internação em 7\% dos casos (incluindo o único que foi a óbito), sendo que aqueles com $\mathrm{FE}>20 \%$ só internaram em 0,8\% das vezes. Estes resultados são compatíveis com relatos que demonstraram que a disfunção ventricular importante aumenta significativamente a incidência de complicações ${ }^{7,10,15}$. A média da fração de ejeção do ventrículo esquerdo no grupo com complicação foi menor, embora essa diferença não tenha sido estatisticamente significativa.

\section{Complicações isquêmicas}

Neste estudo, observamos piora das manifestações isquêmicas coronarianas em 31 (1,6\%) pacientes, sendo que $0,5 \%$ foram importantes (inclusive um óbito). Os preditores de complicações isquêmicas foram lesão do tronco da coronária esquerda, diagnóstico de cardiopatia isquêmica e hipertensão arterial sistêmica ${ }^{16}$. O uso AAS foi associado a complicações isquêmicas somente na análise univariada, provavelmente porque o emprego desta droga é mais freqüente em pacientes com diagnóstico prévio de cardiopatia isquêmica e de mais alto risco. A incidência de complicações isquêmicas importantes em nosso estudo foi de 0,5\%, semelhante aos estudos anteriores, conforme demonstrado na Tabela 5.

\section{Complicações vasculares}

As complicações vasculares (trombose, isquemia, hemorragia) foram as mais incidentes $(3,7 \%)$, sendo que intercorrências leves e complicações moderadas respondem por $3,4 \%$ deste total, o que é semelhante a estudos anteriores ${ }^{1,6-9}$. Os preditores independentes de complicações vasculares foram duração do exame, acesso braquial e uso de anticoagulante. Em relação à técnica utilizada para realizar o procedimento, a maioria dos estudos prévios demonstrou aumento de morbidade e até mortalidade com o uso da técnica de Sones ${ }^{9,11,12,15,19,20}$. Por outro lado, em algumas séries relatadas, observou-se incidência maior de complicações, principalmente importantes, na técnica femoral ${ }^{8,18}$.

\section{Outras complicações}

As complicações vagais ocorreram em 1,9\% dos casos, mas não houve nenhuma complicação importante nesta categoria. As reações vagais, desencadeadas por ansiedade ou dor, surgem atualmente em menor número de casos, possivelmente devido à ampla divulgação da natureza do exame (paciente menos tenso), sedação mais eficaz e maior experiência dos operadores.

As complicações alérgicas ocorreram em 1,5\% dos casos, mas não houve nenhuma complicação importante nesta categoria, o que é semelhante a vários estudos prévios ${ }^{7,12,21,22}$.

As complicações arrítmicas ocorreram em 0,8\% dos casos, sendo que em metade destes a complicação foi importante, necessitando de manobras de ressuscitação ou cardioversão/desfibrilação elétrica. As complicações arrítmicas foram mais freqüentes nas séries de Noto et al. ${ }^{12}$ e de Bono ${ }^{19}$, o que pode estar relacionado à maior gravidade dos pacientes nestes estudos. Neste contexto, a disfunção ventricular esquerda está asso-

TABELA 5

Comparação com estudos prévios

\begin{tabular}{|c|c|c|c|c|c|c|c|c|c|c|}
\hline & Braunwald $^{17}$ & Adams $^{8}$ & CASS $^{9}$ & SCAI $^{10}$ & Wyman $^{6}$ & SCAI $^{11}$ & SCAI $^{12}$ & Nunes $^{7}$ & Oliveira $^{18}$ & Rossato \\
\hline Óbito, \% & 0,14 & 0,45 & 0,20 & 0,14 & 0,12 & 0,10 & 0,11 & 0,50 & 0,09 & 0,05 \\
\hline $\begin{array}{l}\text { Complicação } \\
\text { Importante,\% }\end{array}$ & 3,40 & nd & nd & 1,77 & 2,18 & 1,74 & 1,70 & 3,80 & 1,90 & 1,4 \\
\hline Vascular, $\%$ & nd & nd & nd & nd & 4,00 & nd & nd & 8,80 & 5,71 & 3,7 \\
\hline Importante,\% & nd & 1,44 & 0,70 & 0,57 & 1,60 & 0,46 & 0,43 & 0,50 & 0,63 & 0,3 \\
\hline $\begin{array}{l}\text { Isquêmica } \\
\text { Importante,\% }\end{array}$ & 0,04 & 0,61 & 0,25 & 0,07 & nd & 0,06 & 0,05 & 0,40 & 0,27 & 0,5 \\
\hline $\begin{array}{l}\text { Arrítmica,\% } \\
\text { Importante,\% }\end{array}$ & $\begin{array}{l}\text { nd } \\
1,20\end{array}$ & $\begin{array}{l}\text { nd } \\
1,28\end{array}$ & $\begin{array}{l}\text { nd } \\
\text { nd }\end{array}$ & $\begin{array}{c}\text { nd } \\
0,56\end{array}$ & $\begin{array}{l}1,70 \\
0,30\end{array}$ & $\begin{array}{c}\text { nd } \\
0,47\end{array}$ & $\begin{array}{c}\text { nd } \\
0,38\end{array}$ & $\begin{array}{l}\text { nd } \\
1,40\end{array}$ & $\begin{array}{l}1,54 \\
0,64\end{array}$ & $\begin{array}{l}0,8 \\
0,4\end{array}$ \\
\hline $\begin{array}{l}\text { Embólica,\% } \\
\text { Importante,\% }\end{array}$ & $\begin{array}{l}\text { nd } \\
\text { nd }\end{array}$ & $\begin{array}{l}\text { nd } \\
\text { nd }\end{array}$ & $\begin{array}{l}\text { nd } \\
\text { nd }\end{array}$ & $\begin{array}{l}\text { nd } \\
\text { nd }\end{array}$ & $\begin{array}{c}2,00 \\
\text { nd }\end{array}$ & $\begin{array}{l}\text { nd } \\
\text { nd }\end{array}$ & $\begin{array}{l}\text { nd } \\
\text { nd }\end{array}$ & $\begin{array}{l}0,70 \\
\text { nd }\end{array}$ & $\begin{array}{l}0,27 \\
0,27\end{array}$ & $\begin{array}{c}0,1 \\
0\end{array}$ \\
\hline
\end{tabular}


ciada ao aumento do risco de arritmias durante o cateterismo cardíaco ${ }^{23}$. Já, em diversas outras séries, a incidência de arritmias variou de $0,30 \%$ a $1,4 \%{ }^{6,9-12}$. Embora tenha sido descrito que as bradiarritmias e os distúrbios de condução aumentem pelo uso de contraste iônico ${ }^{24}$, não foi identificada incidência elevada desta complicação em nosso estudo.

Atualmente, existe um grande interesse quanto à segurança do uso de materiais reutilizados, já que as reações pirogênicas estão associadas ao uso de múltiplos cateteres e ao reuso de material, mais do que o material descartável ${ }^{25}$. Em nosso estudo, essa complicação ocorreu em somente $5(0,3 \%$ do total) pacientes, sendo que 3 pacientes apresentaram somente hipertermia e 2, bacteremia, que foi considerada como uma complicação moderada. Embora na classificação adotada as reações pirogênicas e bacteremia estejam incluídas no mesmo tópico, é importante enfatizar a distinção entre estas. As reações pirogênicas são desencadeadas por pirógenos exógenos, produtos de degradação protéica ou substâncias secretadas por bactérias, sendo geralmente auto-limitadas. As bacteremias hospitalares (hemoculturas positivas) são uma síndrome clínica de mais alto risco, também envolvendo aumento do tempo de hospitalização e dos custos hospitalares.

Nossa única complicação embólica foi leve e reversível $(0,1 \%)$. Na literatura, este valor varia de $0,07 \%$ a $2,0 \%{ }^{1,7-9,11,12}$, com comprometimento mais freqüente do sistema nervoso central. A maior incidência ocorre nos valvulopatas, nos procedimentos terapêuticos intervencionistas e não nos diagnósticos. Em três estudos da SCAI ${ }^{10-12}$, AVC ocorreu em 0,07\% dos casos, independentemente da técnica usada. A embolia por colesterol é muito rara e ocorre nos pacientes mais velhos e com mais aterosclerose ${ }^{26}$. Nenhum distúrbio neurológico nos nossos dois casos foi importante.

Os graus máximos de complicações congestivas são o choque cardiogênico e o edema agudo de pulmão, com expectoração hemoptóica, mais propensos de surgir nos pacientes com classe funcional IV, com miocardiopatia e $\mathrm{FE}<30 \%$ ou submetidos a grande sobrecarga de volume no procedimento. Bourassa e Noble $^{15}$ referem incidência de $0,1 \%$. Encontramos, em nossa série, apenas $0,1 \%$ de complicações congestivas, com o choque cardiogênico do único paciente que foi a óbito.

\section{CONCLUSÕES}

Neste estudo, as complicações mais freqüentes do cateterismo cardíaco foram as vasculares, as vasovagais, as isquêmicas e as alérgicas. Estes eventos adversos associaram-se à presença de diabete, tabagismo, cardiopatia isquêmica e lesão do tronco da coronária esquerda, e também naqueles que usavam anticoagulante ou tiveram o tempo de exame aumentado. A incidência de complicações descrita neste estudo foi, geralmente, menor do que àquela em estudos anteriores, com muito baixa mortalidade, o que sugere uma evolução da técnica no decorrer do tempo e reafirma a segurança do procedimento.

\section{REFERÊNCIAS BIBLIOGRÁFICAS}

1. Chandrasekar B, Doucet S, Bilodeau L, Crepeau J, deGuise P, Gregoire J, et al. Complications of cardiac catheterization in the current era: a single-center experience. Catheter Cardiovasc Intervent 2001;52:289-95.

2. Kennedy JW. Complications associated with cardiac catheterization and angiography. Cathet Cardiovasc Diagn 1982;8:5-11.

3. Oliveira EM, Angeli FS, Gottschall CAM. Complicações imediatas do cateterismo cardíaco diagnóstico: um modelo de classificação e estratificação. Rev Bras Cardiol Invas 1997;5:6-10.

4. Judkins MP. Percutaneous transfemoral selective coronary arteriography. Radiol Clin North Am 1968;6:467-92.

5. Grosman W. Complication of Cardiacs Catheterization Incidence, Causes and Presention. In: Grosman W. Cardiac Catheterization and Geography $3^{\underline{a}}$ rb ed. Philadelphia, Lea \& Febiger. 1986:30.

6. Wyman RM, Safian RD, Portway V, Skillman JJ, McKay RG, Baim DS. Current complications of diagnostic and therapeutic cardiac catheterization. J Am Coll Cardiol 1988;12:1400-6.

7. Nunes GL, Nicolela EL Jr, Sousa GM, Maldonado G, Cano MM, Esteves CA, et al. Complicações atuais do cateterismo cardíaco: análise de 1000 pacientes. Arq Bras Cardiol 1991; 56:109-13.

8. Adams DF, Fraser DB, Abrams HL. The complications of coronary arteriography. Circulation 1973;48:609-18.

9. Davis K, Kennedy JW, Kemp HG Jr, Judkins MP, Gosselin AJ, Killip T. Complications of coronary arteriography from the collaborative study of coronary artery surgery (CASS). Circulation 1979;59:1105-12.

10. Kennedy JW. Complications associated with cardiac catheterization and angiography. Cathet Cardiovasc Diagn 1982;8:5-11.

11. Johnson LW, Lozner EC, Johnson S, Krone R, Pichard AD, Vetrovec GW, et al. Coronary arteriography 1984-1987: a report of the registry of The Society for Cardiac Angiography and Interventions I:Results and complications. Cathet Cardiovasc Diagn 1989;17:5-10.

12. Noto TJ Jr, Johnson LW, Krone R, Weaver WF, Clark DA, Kramer JR, et al. Cardiac catheterization 1990: a Report of the Registry of Society of Cardiac Angiography and Interventions (SCAI). Cathet Cardiovasc Diagn 1991;24:75-83.

13. Schroeder SA. The complications of coronary arteriography: a problem that won't go away. Am Heart J 1980;99:139-41.

14. Takaro T, Hultgren HN, Littmann D, Wright EC. An analysis of deaths occurring in association with coronary arteriography. Am Heart J 1973;86:587-97.

15. Bourassa MG, Noble J. Complication rate of coronary arteriography. A review of 5250 cases studied by a percutaneous femoral technique. Circulation 1976;53:106-14.

16. Gordon PR, Abrams C, Gash AK, Carabello BA. Pericatheterization risk factors in left main coronary artery stenosis. Am J Cardiol 1987;59:1080-3.

17. Braunwald E., Swan HJC (Eds). Cooperative Study on Cardiac Catheterization. Circulation 1968; 37 (supl 3): 67-73.

18. Oliveira EM. Complicações imediatas do cateterismo cardíaco diagnóstico em adultos. [Dissertação de Mestrado] Porto Alegre: Fundação Universitária de Cardiologia - Instituto de Cardiologia do Rio Grande do Sul;1997. 86p.

19. Bono D. Complications of diagnostic cardiac catheterization: results from 34.041 patients in the United Kingdom confidential 
enquiry into cardiac catheter complications. The Joint Audit Committee of the British Cardiac Society and Royal College of Physicians of London. Br Heart J 1993;70: 297-300.

20. Cha KS, Kim MH. Feasibility and safety of concomitant left internal mammary arteriography at the setting of the right transradial coronary angiography. Catheter Cardiovasc Interv 2002;56:188-95.

21. Bernardes L, Ramos JM, Quininha J, Figueiredo L, Gonçalves JM, Rato JA. Complicações associadas com a realização de cateterismos cardíacos diagnósticos. Resultados do Laboratório de Hemodinâmica do Hospital de Santa Marta. Rev Port Cardiol 1993;12:851-6.

22. Zimmerman HA, Scott RW, Becker NO. Catheterization of the left side of the heart in man. Circulation 1950;1:357-9.
23. Epstein AE, Davis KB, Kay GN, Plumb VJ, Rogers WJ. Significance of ventricular tachyarrhythmias complicating cardiac catheterization: a CASS registry study. Am Heart J 1990;119(3 pt 1):494-502.

24. Langou RA, Sheps DS, Wolfson S, Cohen LS. Intraventricular conduction during coronary arteriography in patients with pre-existing conduction abnormalities. Invest Radiol 1977; 12:505-9.

25. Kundsin RB, Walter CW. Detection of endotoxin on sterile catheters used for cardiac catheterization. J Clin Microbiol 1980;11:209-12.

26. Rosman HS, Davis TP, Reddy D, Goldstein S. Cholesterol embolization: clinical findings and complications. J Am Coll Cardiol 1990;15:1296-9. 